\title{
Behavioral states may be associated with distinct spatial patterns in electrocorticogram
}

\author{
Heracles Panagiotides - Walter J. Freeman • \\ Mark D. Holmes · Dimitrios Pantazis
}

Received: 30 December 2009/Revised: 13 October 2010/Accepted: 25 October 2010/Published online: 13 November 2010

(C) The Author(s) 2010. This article is published with open access at Springerlink.com

\begin{abstract}
To determine if behavioral states are associated with unique spatial electrocorticographic (ECoG) patterns, we obtained recordings with a microgrid electrode array applied to the cortical surface of a human subject. The array was constructed with the intent of extracting maximal spatial information by optimizing interelectrode distances. A 34-year-old patient with intractable epilepsy underwent intracranial ECoG monitoring after standard methods failed to reveal localization of seizures. During the 8-day period of invasive recording, in addition to standard clinical electrodes a square $1 \times 1 \mathrm{~cm}$ microgrid array with 64 electrodes (1.25 mm separation) was placed on the right inferior temporal gyrus. Careful review of video recordings identified four extended naturalistic behaviors: reading, conversing on the telephone, looking at photographs, and face-to-face interactions. ECoG activity recorded with the microgrid that corresponded to these behaviors was collected and ECoG spatial patterns were analyzed. During periods of ECoG selected for analysis, no electrographic seizures or
\end{abstract}

H. Panagiotides $(\bowtie)$

Department of Neurological Surgery, University of Washington, 1959 Pacific Ave, Seattle, WA, USA

e-mail: hercp@u.washington.edu

W. J. Freeman

Department of Molecular \& Cell, Division of Neurobiology,

University of California, Donner 101, Berkeley, CA, USA

\section{D. Holmes}

Regional Epilepsy Center, Department of Neurology, University of Washington, Harborview Medical Center, 325 Ninth Ave, Seattle, WA, USA

\section{Pantazis}

Signal \& Image Processing Institute, Department of Electrical Engineering, University of Southern California, 3740

McClintock Ave, Los Angeles, CA 90089-2564, USA epileptiform patterns were present. Moments of maximal spatial variance are shown to cluster by behavior. Comparisons between conditions using a permutation test reveal significantly different spatial patterns for each behavior. We conclude that ECoG recordings obtained on the cortical surface with optimal high spatial frequency resolution reveal distinct local spatial patterns that reflect different behavioral states, and we predict that similar patterns will be found in many if not most cortical areas on which a microgrid is placed.

Keywords Neural circuits - Behavioral states . Perception $\cdot$ EEG $\cdot$ Epilepsy $\cdot$ Spatial EEG patterns

\section{Introduction}

The aim of our project was to seek and measure spatial patterns of cortical electrical activity that we could correlate with intentional cognitive behaviors. Our choice of the electrocorticogram $(\mathrm{ECoG})$ was based on prior success in animal studies. Spatial patterns were extracted from multichannel ECoGs recorded from the sensory and limbic cortices of rabbits (Barrie et al. 1996), cats (Freeman et al. 2003c) and gerbils (Ohl et al. 2001) with high-density planar arrays of electrodes fixed on the pial surfaces. Reinforcement training shaped the patterns, so that they could be classified with respect to the conditioned stimuli (CS) eliciting them. The choice of ECoG rather than local field potentials (LFP) and units from arrays of depth electrodes (Ghovanloo et al. 2003; Pang et al. 2005) was based on ease of surgical placement, immunity from tissue damage by avoiding insertions, and long-term stability of signals. An additional advantage was the fact that the ECoG contained only contributions from neuron populations generating open 
fields, whereas the LFP contained far more complex mixtures of open and closed fields (Chap. 4 in: Freeman 1975).

The most important experimental parameter to set in the search for classifiable spatial patterns in the ECoG was the distance between the electrodes in the array. The search for spatial patterns differed from the search for temporal signals. The main requirement for identifying a temporal signal was that the digitizing rate had to be high enough to capture the highest temporal frequency components, thereby avoiding undersampling and aliasing. The requirement for measuring the spatial patterns was sampling at intervals sufficiently small to avoid aliasing but large enough to avoid oversampling. The criterion for an optimal interval was based on calculation of the spatial power spectral density $\left(\mathrm{PSD}_{\mathrm{X}}\right)$ of human ECoG (Freeman et al. 2000) from 64 ECoG signals recorded with a $64 \times 1$ linear array on the superior temporal gyrus $(0.5 \mathrm{~mm}$ spacing, $32 \mathrm{~mm}$ length, giving a spectral range from 0.016 to $1 \mathrm{cycle} / \mathrm{mm}$ ). The $\mathrm{PSD}_{\mathrm{X}}$ showed a power-law (linear) decrease in log power with increasing log spatial frequency up to a spatial frequency of 0.4 cycles $/ \mathrm{mm}$, above which the spectrum was flat. Activity above that inflection was treated as noise. By the Nyquist criterion the optimal sampling frequency was 0.8 cycles $/ \mathrm{mm}$, giving a spacing of $1.25 \mathrm{~mm}$. An $8 \times 8$ array was $10 \times 10 \mathrm{~mm}$ in width, which fit onto many single gyri in the human brain without bridging a sulcus.

The performance of the microgrid in extracting spatial ECoG patterns was tested thoroughly in recordings from sensory cortices of animals that were trained to discriminate olfactory (Freeman and Grajski 1987), visual, auditory, and somatic CS (Freeman and Barrie 2000; Freeman and Rogers 2002). Spatial patterns of amplitude were shown to be classifiable with respect to conditioned stimuli in both classical and operant conditioning (Freeman and Viana Di Prisco 1986). The spatial patterns were found to occur in brief segments lasting 80-120 ms, which had high amplitude and high spatial variance of a common waveform but minimal temporal and spatial variance of the frequency of the waveform (Freeman 2004a, b). The classification was by measuring the 64 amplitudes of the common waveform in each frame, constructing a $64 \times 1$ feature vector for each frame, expressing the vector as a point in 64-space, and forming clusters of points from multiple trials with each discriminated stimulus. Classification was by dividing data sets into a training set to locate centers of gravity of clusters and a test set to classify feature vectors by the Euclidean distance to the closest center of gravity.

The goodness of classification was reduced or abolished by randomization of the data: spatial scrambling of channels, temporal scrambling of phase values, and most effectively by random shifts of ECoG segments in the 64 samples from each frame (Freeman 2005). The classificatory information was solely in the amplitudes of the signals, not in the frequencies. Every frame had the same waveform with the same carrier frequency but with spatial amplitude modulations of the amplitudes and phases. The effectiveness of the microgrid for extracting the spatial differences of both the amplitude and phase of the high-frequency components of the ECoG was measured by calculating the point spread function (PSF). This was the bell-shaped surface distribution of dendritic potential above an active point in cortex) that was predicted for activation of a single dendrite or a cortical column (Fig. 2 in: Freeman 2006). The results simulated the full range of observed spatial differences in amplitude modulation of the shared waveform.

The properties of the phase of the ECoG that were derived from animals were replicated in the analysis of ECoG from a human subject (Freeman et al. 2006a, b). In particular, the radial phase gradients ('phase cones') were readily documented, showing that the spatial resolution provided by the grid would be adequate for classification of amplitude patterns. The principal difficulty encountered was the definition of an appropriate set of behaviors. The animal studies were designed to study the ECoGs of sensory cortices, with the exception of a study in cats, in which three $4 \times 4$ microgrids were fixed on the visual, auditory and somatic cortices. Two electrodes were placed in the olfactory bulb, and a $2 \times 8$ array was fixed on the entorhinal cortex, taking advantage of the flat surface of the bony tentorium that underlay the limbic cortex (Freeman et al. 2003c). That study revealed global amplitude patterns with an intermittently shared waveform. The goodness of the classification was maximal when all 64 channels were used. The goodness declined significantly when any component was removed, showing that widely separated areas of the forebrain synchronized repeatedly at frame rates in the theta range (Freeman and Burke 2003) and sustained classifiable spatial patterns. This result encouraged us to search for classifiable amplitude patterns in the temporal lobe. The lack of dependence of classification on ECoG frequencies led us to adopt the magnitude of the spatial variance of amplitude as our criterion for selection of potentially classifiable ECoG segments.

The placement of the human microgrid onto the inferior temporal gyrus, far from any sensory or motor area, was dictated entirely by neurosurgical considerations for the welfare of the patient, who gave informed consent for the recordings. This region has rich interconnections with both the amygdala and the orbital frontal cortex through the uncinate fascicule. It also sends projections to the hypothalamus and receives inputs from the three sensory systems in the temporal lobes, the visual, the auditory and the olfactory systems as well as from the insula (gestation and awareness of internal physiological states. The temporal pole has been implicated in social behavior. Lesions of the temporal pole that spared the amygdala in female monkeys 
resulted in inappropriate vocal and facial social signals, inability to recognize social signals of other monkeys, lack of interest in social peer monkeys lack of aggression and neglect of their infants (Bucher et al. 1970; Kling and Steklis 1976; Kling et al. 1993; Olson et al. 2007).

In humans, socioemotional deficits have been associated with temporal pole damage, such as the Kluver-Bucy syndrome that can result from herpes encephalitis affects the medial and anterior temporal lobes (Lilly et al. 1983). Similar symptoms can be observed following anterior temporal lobe lesions that result from epilepsy (Anson and Kuhlman 1993). In patients with temporal variant of frontal temporal dementia and only right temporal pole atrophy personality and social behavior deficits are observed, such as loss of social dominance, lack of empathy and neurotic behavior(Mummery et al. 2000). Temporal pole damage can lead to unpredictable mood states, rapidly cycling bipolar disorder (Murai and Fujimoto 2003) and cycling levels of depression, anxiety and irritation (Glosser et al. 2000).

The temporal poles have also been implicated in face processing. Although the fusiform gyrus (Grill-Spector et al. 2004; Kanwisher et al. 1997, 1999; Kanwisher and Yovel 2006) and the superior temporal sulcus (Gross 1992; Perrett et al. 1985) are the structures mostly known for their involvement in the perception of face identity and expression, there evidence suggesting that the temporal poles are also involved in face processing. Bilateral and even unilateral lesions of the temporal poles can produce a form of prosopagnosia that is not accompanied by generalized memory failure (Damasio et al. 1990). Cognitive deficits of this "amnesic associative prosopagnosia" also include voice and gait mediated recognition (Olson et al. 2007). Left temporal pole lesions cause impairments in naming famous faces (Glosser et al. 2000), while atrophy of this region produces progressive prosopagnosia (Thompson et al. 2003). With electrodes implanted in the right ventral temporal pole ERP components with latencies around $350 \mathrm{~ms}$ were recorded (Allison et al. 1994, 1999; McCarthy et al. 1999; Puce et al. 1999). Finally, imaging studies have revealed that the temporal pole is involved in person (not face) recognition and the appraisal of facial emotional expressions (Damasio et al. 1990).

The temporal pole has also been implicated in tasks involving theory of mind (inferences about the intensions, desires and beliefs of others). Functional neuroimaging studies have revealed activation during theory of mind tasks such as thinking of other people's emotions, making moral decisions or judging intentionality in viewing geometric shapes that move around. Evidence comes from neuroimaging studies and patients with temporal pole lesions who exhibit lower levels of empathy (Olson et al. 2007).
Given the broad involvement of the temporal lobe in cognitive and social behaviors, and in consideration of the comfort and convenience of the patient, we did not proceed to a behavioral design with double blind controls. Rather, we sought the amplitude correlates of the ECoG in four behaviors that the patient engaged in repeatedly: (face-toface interaction, looking at pictures, reading, speaking on the telephone). Pattern localization was based on local maxima in the spatial variance of the microgrid electrical activity; pattern classification was based on the feature vectors at the maxima. Principal components analysis and a permutation test revealed significant pattern differences between behavioral conditions. Our aim was to demonstrate the existence of classificatory information relating to cognition in the human ECoG at the high spatial frequencies that have heretofore been neglected.

\section{Methods}

\section{Subject}

The subject, a 34-year-old woman with a history of intractable partial complex seizures, had subdural strip electrodes placed over lateral and basal-temporal regions for preoperative evaluation prior to surgical intervention. In addition to the clinical electrodes, a high-density electrode microgrid through a right temporal burr hole was placed onto the anterior surface of the right inferior temporal lobe gyrus. The patient underwent continuous ECoG and behavior monitoring for 8 days; the length of recording time was determined solely by clinical consideration. The microgrid remained in place during the entire 8-day period, as did the clinical electrodes. No complications were observed during the procedure. The data were collected in the EEG and Clinical Neurophysiology laboratory of Harborview Hospital, University of Washington in Seattle. Data collection and management were in agreement with the protocol approved by the University's Human Subjects Review Committee. The patient gave informed consent for the placement and use of the clinical electrodes and the microgrid.

\section{Electrode placement}

Fifty-two intracranial subdural clinical electrodes were placed over lateral and basal temporal regions of both hemispheres through temporal burr holes. Additionally, a microgrid array of $8 \times 80.5 \mathrm{~mm}$ stainless steel wires spaced $1.25 \mathrm{~mm}$ apart was placed over a $1 \mathrm{~cm}^{2}$ cortical patch on the right anterior inferior temporal gyrus (Fig. 1). The interelectrode dimensions of the microgrid were established on the basis of knowledge of the spatial 
Fig. 1 Left Location of microgrid in a radiograph. Right Drawing of microgrid and its electrode arrangement

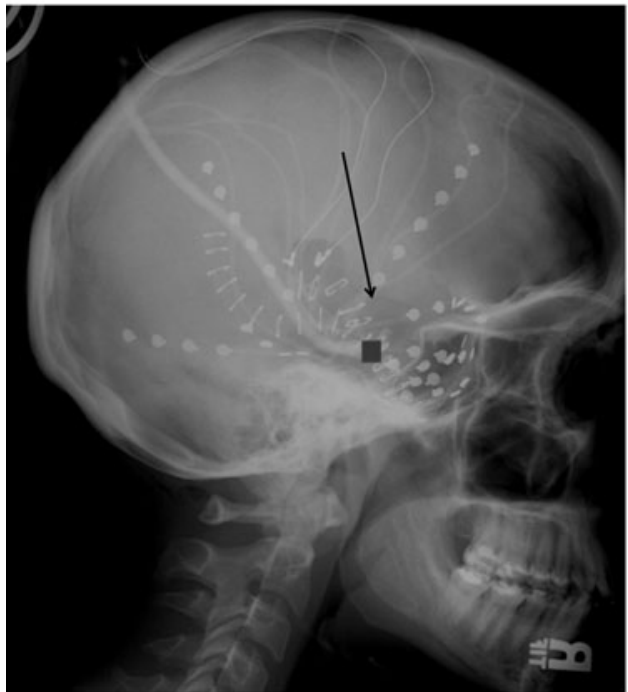

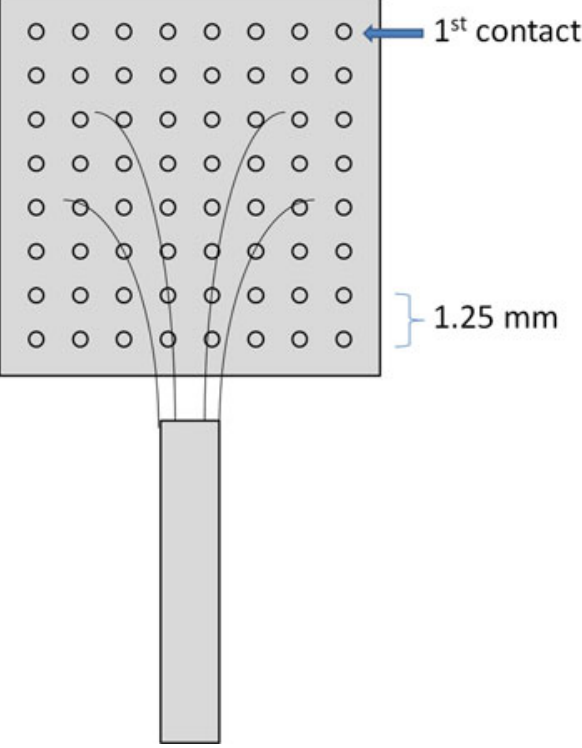

frequency spectrum of human EEG from the cortical surface (Freeman et al. 2000). More details of the electrode placement and microgrid design are available in Freeman et al. 2006a, which uses the same experimental data but a different set of behavioral moments, in order to investigate the power spectral density and phase of brain signals.

\section{Data collection}

The EEG was amplified with a Nicolet BMSI 5000 system having a fixed gain of 1628 and analog filters set at $0.5 \mathrm{~Hz}$ high pass and $120 \mathrm{~Hz}$ low pass. The ADC gave 12 bits with the least significant bit of $0.9 \mu \mathrm{V}$ and maximal range of $\pm 2048 \times 0.9 \mu \mathrm{V}$. The reference and ground placements for all 64 electrodes were, respectively, the $\mathrm{Cz}$ and $\mathrm{Pz}$ scalp locations [midline frontal and parietal sites in the standard 10-20 clinical system (Reilly 1999). The Nicolet system digitized data at $420 \mathrm{~Hz}$ and down-sampled to $200 \mathrm{~Hz}$. Off-line low pass filtering at or below $55 \mathrm{~Hz}$ was performed to minimize $60 \mathrm{~Hz}$ artifact.

Selection of behavior moments

The entire video record of the patient during the 8 days of hospitalization was reviewed. Sustained behaviors involving visual and auditory social interactions and reading were sought and identified. Four behaviors were selected: (a) face to face interaction with another person $(5 \mathrm{~min})$, (b) looking at pictures $(27 \mathrm{~min})$, (c) reading a book or a magazine (10 min), and (d) speaking on the telephone (14 min). The selection of these behaviors was motivated by the function of the location over which the microgrid was placed. As reviewed in the introduction, the temporal pole is implicated in both visual and auditory social behavior, and particularly with face processing. This region is believed to be active during "theory of mind" tasks. The inferior temporal lobe in associated with the integration of higher processing of complex stimuli.

\section{Preprocessing}

Only the microgrid physiological record during the aforementioned behaviors was considered for analysis. The record during those time periods was seizure-free. Unlike conventional EEG recordings, corticographic recordings are less susceptible to muscle artifacts, such as eye blinks, body, head and tongue movement, than conventional EEG recordings. The main artifact that may be seen with intracranial EEG is pulse signals, if the electrode is near a vessel. Other artifacts include the electrode wire being grossly pulled from the outside, which is a rare occurrence because the subdural electrodes are securely anchored (Spencer et al. 2008). In this study, the physiological record for each condition was visually inspected and motion and other artifacts were identified and demarcated. Artifacts were characterized by their amplitude and overall morphology. The most common artifact was signals sharply deviating from the overall norm in one or more electrodes. Figure 2 shows examples of such artifact segments. Overall, the percentage of the data that were excluded from analysis was: $24.65 \%$ for the face condition, $24.8 \%$ for the pictures condition, $0.3 \%$ for the reading condition, and $14.7 \%$ for the phone condition. All artifact-free segments of each specific behavior were entered into subsequent analysis. 
Fig. 2 Sample segments excluded from analysis due to artifacts
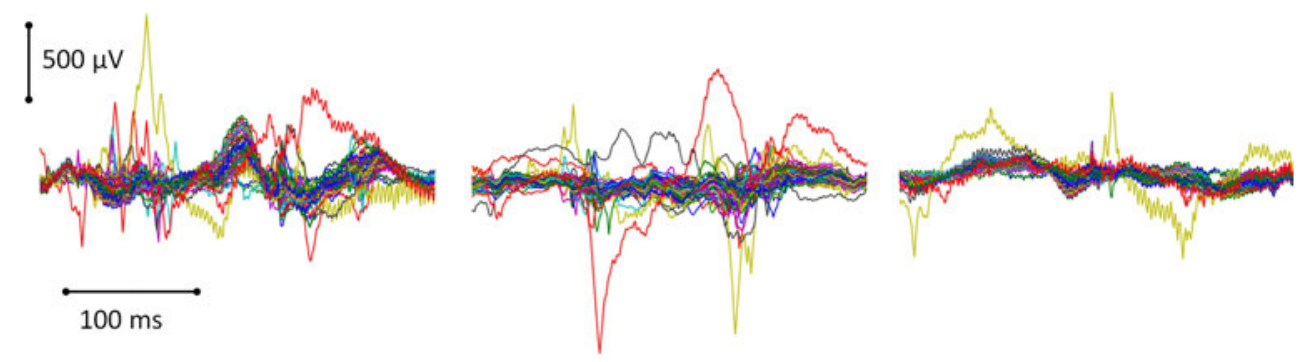

Fig. 3 Left Selection of time points for data analysis. Spatial standard deviation $\sigma(t)$ is computed for every time point $(t \in S)$ and a threshold of $35 \mathrm{mV}$ is used to identify time regions of high spatial variance. Only time points corresponding to local maxima $\left(t \in S_{M}\right)$ within these regions are considered for further analysis (points marked with circles). Right Histograms of spatial standard deviation for each condition
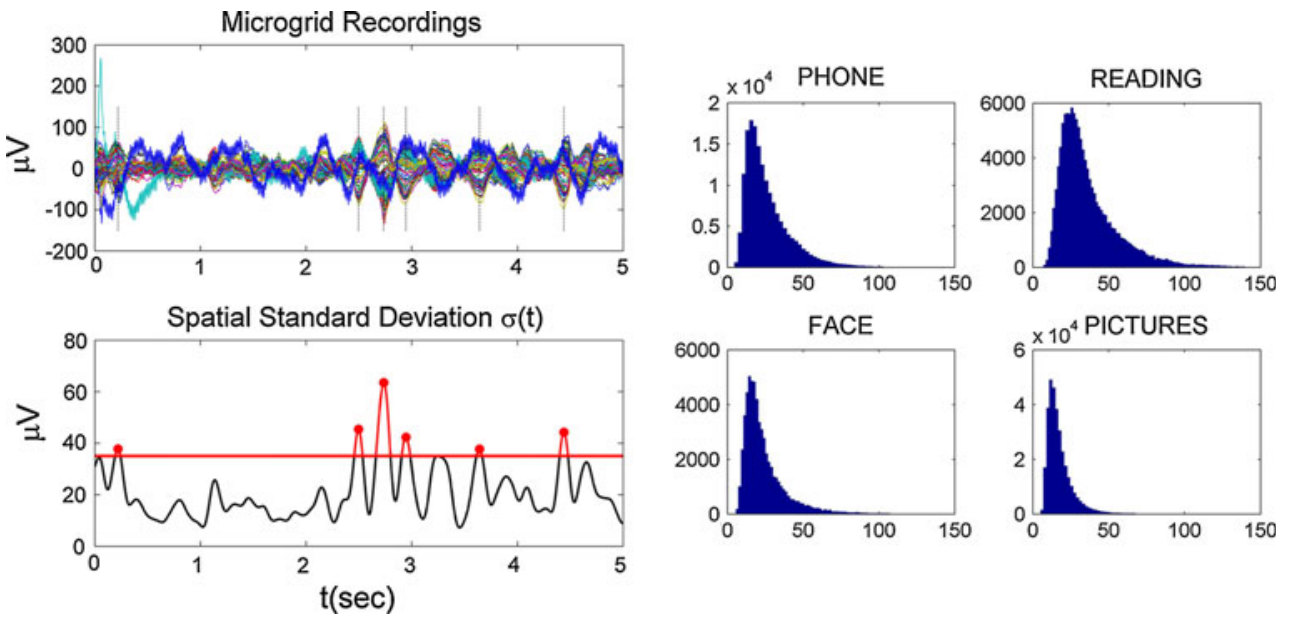

\section{Spatial variance}

The spatial variance $\sigma^{2}(t)$ based on the voltage values at the microgrid electrodes was computed for each time point ${ }^{1}$ :

$\sigma^{2}(t)=\frac{\sum_{i=1}^{N}\left(v_{i}(t)-\bar{v} .(t)\right)^{2}}{N-1}, \quad t \in S$

where $v_{i}(t)$ is the voltage at electrode $i$ at time $t, N=64$ is the total number of electrodes, and $S$ is the artifact-free recording time period for each condition. Time segments with large spatial standard deviation $(\sigma(\mathrm{t})>35 \mathrm{mV}$, a threshold that allows enough trials per condition) were selected (Fig. 3). Among these time segments, only the time points with the local maximum variance were kept for subsequent analysis $\left(\mathrm{t} \in S_{M}\right.$, where $S_{M}$ is the set of time points with local maximum variance), leading to a total of $T_{M}=1138$ time points for speaking on the phone, 1347 for reading, 199 for face-to-face interaction, and 254 for looking at pictures. Each one of these time points consisted of an $8 \times 8$ matrix of voltage values, therefore each condition yielded a set of $8 \times 8$ matrices.

Principal ECoG components of conditions

Each group of $8 \times 8$ matrices of voltage values was separately subjected to principle component analysis (PCA), in

1 The bar indicates averaging over the dotted subscript. order to visualize the main components of the data. For this purpose, the $8 \times 8$ measurement matrices were reshaped into $\mathrm{N} \times 1$ vectors $\boldsymbol{v}(\boldsymbol{t})$ and concatenated (after being transposed) into a voltage matrix $\mathbf{V}$ with dimension $T_{M} \times N$, where $T_{M}$ is the number of time points with maximum variance for each condition (cardinality of $S_{M}$ ) and $N=64$. We applied PCA on matrix V. PCA first centers the data by making each variable (electrode in our case) zero mean. We denote the centered data as $\boldsymbol{v}^{\boldsymbol{o}}(t)=$ $\boldsymbol{v}(t)-\overline{\boldsymbol{v}}($.$) and \boldsymbol{V}^{\mathbf{o}}$. PCA produced $N$ principal component vectors $\mathbf{p c}_{j}, j=1 \ldots N$, as well as the projections of the centered voltage vectors $\boldsymbol{v}^{\mathbf{o}}(t)$ on the principal components, called scores:

$\boldsymbol{v}^{\boldsymbol{o}}(t)=\sum_{j=1}^{N} \operatorname{score}_{t} \mathbf{p} \mathbf{c}_{j}, \quad t \in S_{M}$

or in matrix form:

$V=\mathbf{S C O R E} \cdot \mathbf{P C}^{\mathbf{T}}$

where SCORE is a $T_{M} \times N$ matrix, and PC is a $N \times N$ matrix with each column $j$ populated by the principal component $\mathbf{p c}_{j}$.

Multiple discriminant analysis

To investigate whether the recorded signals on the microgrid vary depending on the condition, we applied multiple 
discriminant analysis (MDA), which is a generalization of the Fisher Linear Discriminant analysis for multiple classes (Duda et al. 2000, p. 121). Our data comprises $c=4$ classes, which correspond to 4 conditions: Phone, Reading, Face, Pictures. While PCA seeks directions that are efficient for representation of the data, MDA seeks directions that are efficient for discrimination. In particular, it finds weight vectors $\boldsymbol{w}_{k}, k=1 \ldots c-1$, which linearly transform the data into new measurements $y_{k t}=\boldsymbol{w}_{k}^{T} \boldsymbol{v}(t)$ that are maximally separable among the classes.

To explore whether differences across conditions are attributed to topographical changes in voltage rather than changes of the overall voltage level, we normalized the voltage measurements $v_{i}(t)$ into zero mean unit variance across electrodes before being subjected to MDA:

$v_{i}^{N}(t)=\frac{v_{i}(t)-\bar{v} \cdot(t)}{\sigma(t)}$

\section{Permutation test}

To identify the spatial profile of the electrodes that differ among conditions, we used a permutation test (Pantazis et al. 2005). The permutation method does not assume any parametric distribution (for example normally distributed samples), and automatically corrects for multiple comparisons. We used a t-statistic contrast between conditions, which allows for different number of samples and different variance for each condition.

Assume a total of $T_{A}$ voltage measurements $v_{i}^{A}(t)$ and $T_{B}$ voltage measurements $v_{i}^{B}(t)$ for two conditions $\mathrm{A}$ and $\mathrm{B}$, respectively. We calculate a $t$-statistic map $t_{i}, i=1 \ldots N$, by taking the difference of the means across time between the two conditions and dividing by the standard deviation:

$t_{i}=\frac{\bar{v}_{i}^{A}(.)-\bar{v}_{i}^{B}(.)}{\sigma_{i}^{A B}}$

$\sigma_{i}^{A B}=\frac{\left(T_{A}-1\right) \sigma_{i}^{A}+\left(T_{B}-1\right) \sigma_{i}^{B}}{T_{A}-T_{B}+2}$

where $\sigma_{i}^{A}$ and $\sigma_{i}^{B}$ are the standard deviations of the voltage measurements at electrode $i$ across time. Equation 6 allows for different number of samples and variances for each group. To detect electrodes that demonstrate statistical significant difference across conditions, we need to threshold the statistical map $t_{i}$, while controlling for multiple comparisons.

We assume the voltage measurements were exchangeable, i.e. we can permute the matrices between the two conditions, under the null hypothesis of no condition effect. For every permutation sample, we exchange voltage measurements between the two conditions to create $v_{i}^{A *}(t)$ and $v_{i}^{B *}(t)$, while keeping the number of observations for each group the same as the original data. The star $(*)$ indicates that variables are produced by permutation. We then estimate the permutation statistic:

$t_{i}^{*}=\frac{\bar{v}_{i}^{A *}(.)-\bar{v}_{i}^{B *}(.)}{\sigma_{i}^{A B *}}$

By repeating this process multiple times, we create $M=100000$ permutation samples (in one of them we keep the original set), where each time we randomly permute the matrices between the two conditions. To control the family-wise error across all electrodes, we use the maximum statistic approach, which computes the maximum absolute value of the t-statistic for each permutation sample:

$t_{\max }^{*}=\max _{i}\left(\left|t_{i}^{*}\right|\right)$

We use the M permutation samples to build the empirical distribution of $t_{\max }^{*}$ and then define a threshold that leaves $\alpha=5 \%$ of the distribution on the right side. By applying this threshold to the t-statistic matrix of the original data, we identify the electrodes that have statistical significant effects while controlling the family-wise error among all spatial locations. Figure 4 schematically outlines the methodology of this portion of the analysis.

\section{Results}

Principal ECoG components of conditions

Microgrid measurements with the highest spatial variance were grouped by condition and separately analyzed with principal component analysis as described in the "Method" section (Eq. 2). Figure 5 shows scatter plots of the scores for the first two principal components (score ${ }_{t, 1}$ and score $t_{t, 2}$, $t \in S_{M}$ ) for each condition. In other words, it displays the projections (inner product) of each microgrid measurement $\boldsymbol{v}(t)$ on the first two principal components $\mathbf{p c}_{1}$ and $\mathbf{p} \mathbf{c}_{2}$.

For all conditions, the scatter plots form two clusters approximately symmetric around the center $(0,0)$. The bottom right clusters have 559 points for speaking on the phone, 597 for reading, 93 for face-to-face interaction, and 120 for looking at pictures. The top left clusters have 579 points for speaking on the phone, 750 for reading, 106 for face-to-face interaction, and 134 for looking at pictures.

To focus on topographic patterns rather than voltage level differences, we normalized the voltage measurements into zero mean and unit variance across electrodes, as in Eq. 4. Then, we averaged the microgrid measurements corresponding to each cluster separately. Figure 6 shows the average topographic voltage maps for the bottom right clusters (first row), top left clusters (second row), and their summation (third row). The average topographic voltage maps revealed that the two distributions are symmetrically 

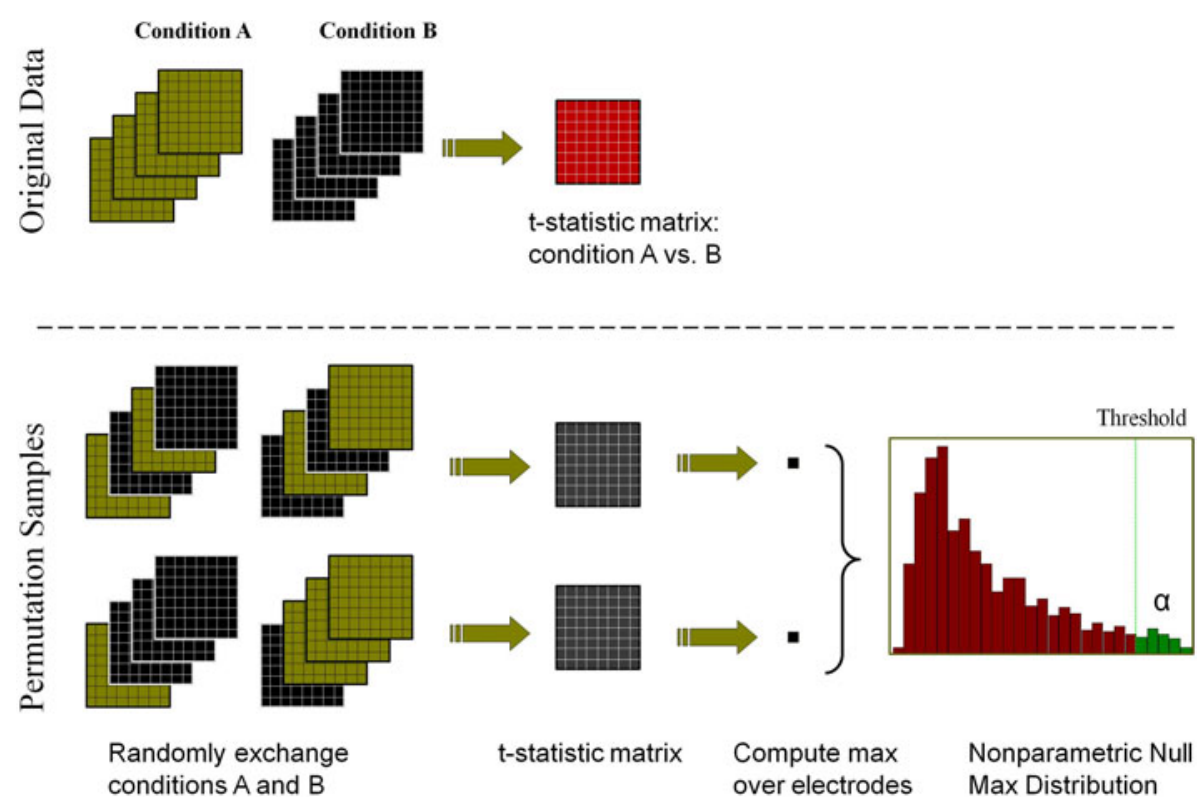

Fig. 4 Illustration of the permutation method: the original voltage matrices produce many permutation samples by exchanging Condition A and Condition B labels. The matrices are then averaged and normalized with the standard deviation to produce t-statistic matrices (maps $t_{i}^{*}$ from Eq. 7) separately for each permutation sample. We compute the maximum absolute t-statistic over all electrodes for each permutation sample ( $t_{\max }^{*}$ from Eq. 8) and use it to estimate the empirical distribution of the maximum statistic. The value that leaves $\alpha=5 \%$ of the distribution on the right side is the threshold that is then applied to the t-statistic matrix of the original data (map $t_{i}$ from Eq. 5). Values above the threshold are significantly different between the two conditions. The procedure guarantees that the family-wise error rate is exactly controlled at level $\alpha$
Fig. 5 Scatter plots of the scores ( score $_{t, 1}$ and score s. $_{t, 2}$ ) of each voltage vector $\boldsymbol{v}(t)$ for the first two principal components. Only time points of maximum local variance were analyzed $\left(t \in S_{M}\right)$, separately for each condition
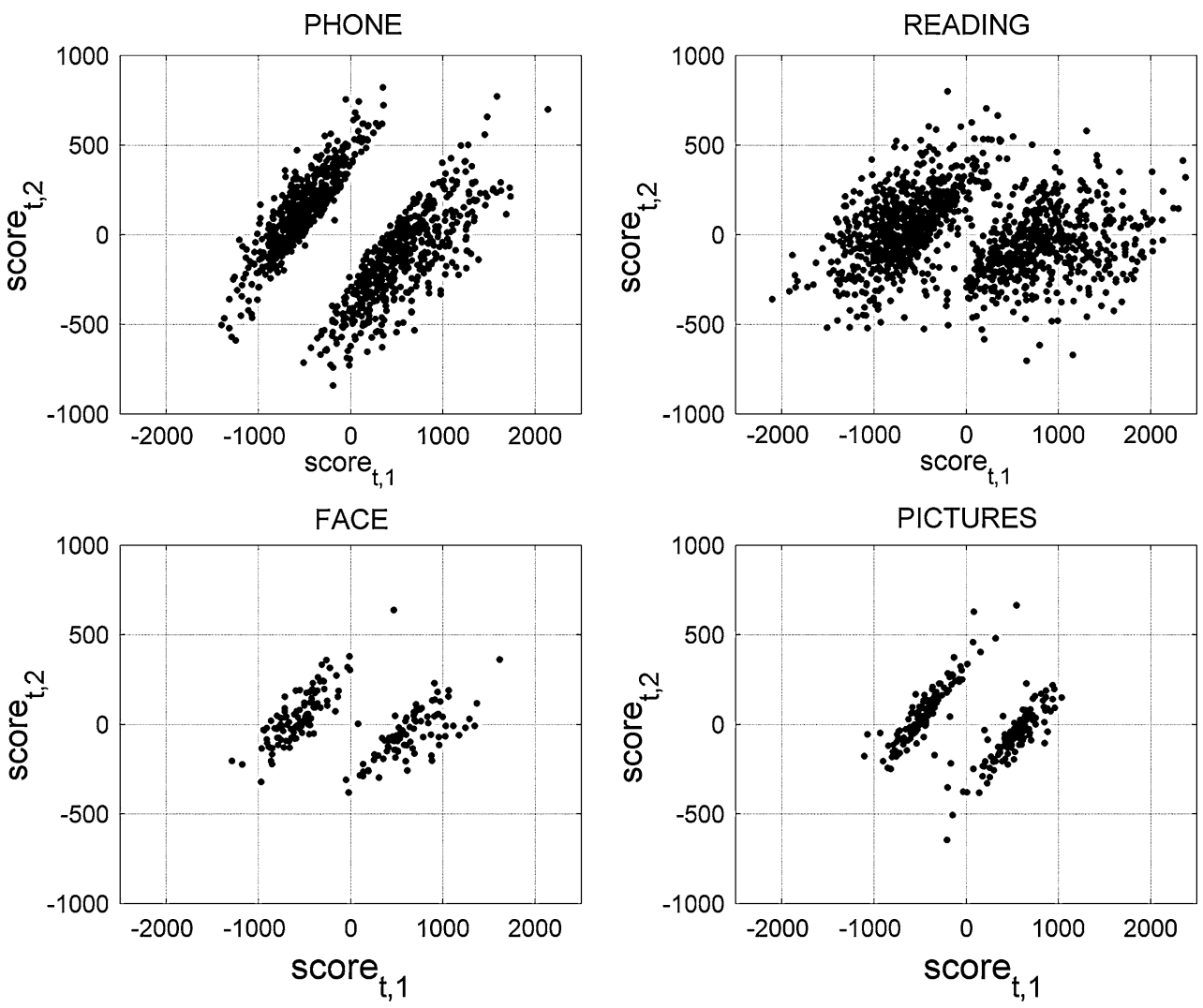
opposite to each other in each condition. Also, despite the great resemblance of topographies across conditions, differences are still visible. Our MDA and permutation test analysis explored whether these differences are significant.

Multiple discriminant analysis

We performed MDA analysis only on topographies corresponding to the bottom right cluster of Fig. 5. Joint analysis of both clusters would hinder statistical analysis, since clusters are far apart and located in similar areas for all conditions. Furthermore, voltages were normalized as in Eq. 4.

Figure 7 shows scatter plots of MDA coefficients $y_{k t}$, $k \in\{1,2,3\}, t \in S_{M}$, bottom right cluster. All four conditions are clearly separable, indicating that microgrid voltage measurements recording above $1 \mathrm{~cm}^{2}$ of cortex have distinct topographies per condition.

\section{Inferential statistical comparisons}

Between-condition analysis of the matrices at the local maxima of the spatial variance was done using a permutation test, as described in the "Method" section. Similarly to MDA analysis, only the bottom right clusters of Fig. 5 were analyzed, and voltage measurements were normalized into zero mean unit variance as in Eq. 4.

The results for each condition comparison are shown in Fig. 8. Significant electrodes (corrected for multiple comparisons) are displayed on top with white color. Different sets of electrodes were significant for each pair of conditions. The t-statistical maps $t_{i}$ of the original data, estimated using Eq. 5, are displayed at the bottom of Fig. 8.

\section{Discussion}

We have detected and analyzed local spatial ECoG patterns registered by a high-density array during naturalistic behaviors. These topographic patterns appear very similar across conditions, as shown in Fig. 6 (first and second row). However, further investigation with multiple discriminant analysis demonstrated that the topographic patterns are different between conditions. A permutation analysis further localized these differences on various sets of electrodes across the microgrid. Depending on the conditions being contrasted, different sets of electrodes were significantly different. Therefore, the local maxima of spatially variant activity revealed topographic patterns that significantly differed between conditions of naturalistic behaviors.

The present experiment was designed to test the prediction, which was based on animal studies of ECoG, that textured high spatial frequency information would be found in human ECoG that was correlated with cognitive behaviors. The location of the microgrid was based on proximity to an expected pathological focus, but it was random with respect to an area engaged in a certain cognitive function. Estimates of the surface area of human cerebral cortex range from 1640 to $2500 \mathrm{~cm}^{2}$, with $22 \%$ in the temporal lobe (Nieuwenhuys et al. 1998; Henery and
Fig. 6 Average topographic distributions of normalized voltages $v_{i}^{N}(t)$ across the microgrid. First row average of topographies for time points corresponding to the bottom right clusters of Fig. 5, separately for each condition. Second row average of top left clusters of Fig. 5. Third row summation of above.

Topographies on first and second rows are nearly opposite. Also, there is great similarity of topographies between conditions, but also visible differences
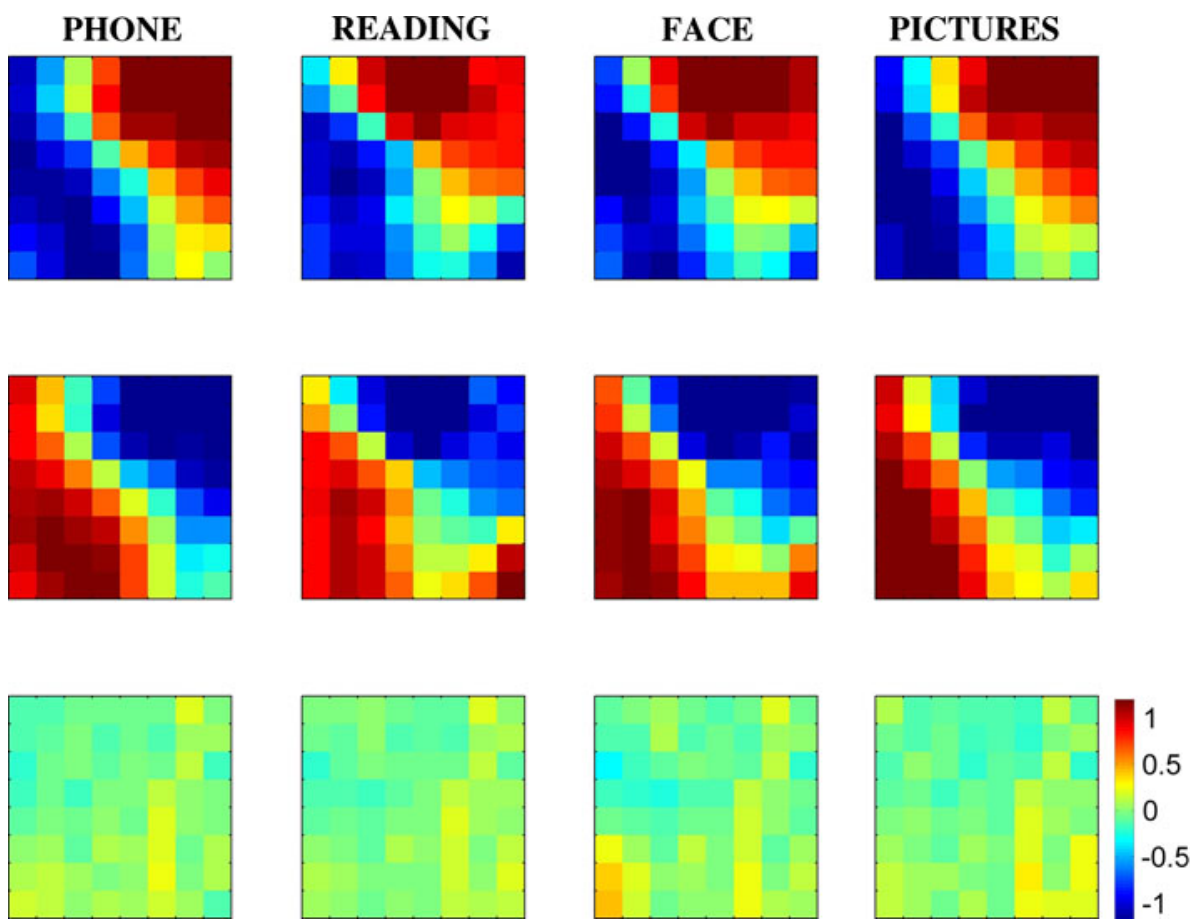
Fig. 7 Scatter plots of the first 3 linear discriminant coefficients $y_{k t}$, for voltage measurements corresponding to the bottom right clusters of

Fig. 3 ( $t \in S_{M}$, bottom right cluster). All four conditions are clearly separable
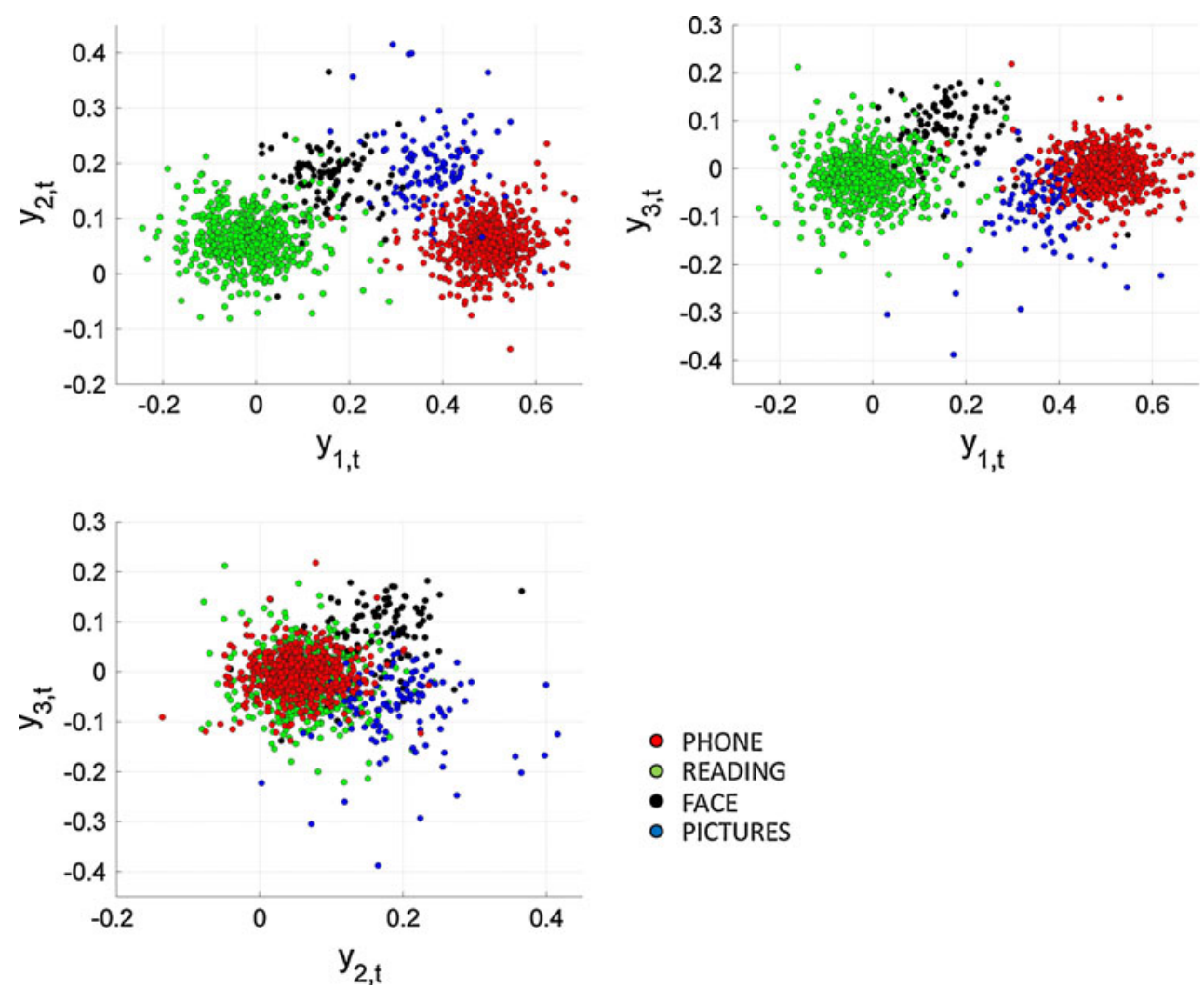

Mayhew 1989; Tramo et al. 1995). The remarkable finding of behavioral correlates in a sample size of $1 \mathrm{~cm}^{2}(1 \%$ of the temporal lobe, $0.05 \%$ of the cortex) lead us to predict that such information will be found in many, if not most, cortical locations, and could have an effect on applications, such as the design of brain-computer interfaces. Our prediction is supported by the global phase-locked pattern correlates of CS found in cats (Freeman and Burke 2003), and by the demonstration of classifiable spatial patterns of beta activity in the scalp EEG (Ruiz et al. 2009) recurring in phase-locked segments recurring at rates in the theta range (Freeman et al. 2003a, b; Ruiz et al. 2007; Pockett et al. 2009).

We have observed two distinct voltage topographies, opposite to each other (Fig. 6). Such brain responses are often reported in literature. For example, in EEG/MEG brain responses are denoted as positive or negative, with the letters $\mathrm{P}$ and $\mathrm{N}$, followed by the time delay in milliseconds, such as P20, N30 etc. An example is a study of median nerve stimulation, which yielded a number of these components with inverted topographies (Allison et al. 1991). Dipolar current source-sink pairs that inverted potentials from dendritic current flow can cause such responses. We postulate that a similar phenomenon occurs close to the microgrid. Current dipoles in the vicinity of the microgrid produce electric currents with alternating phases, therefore causing the two spatial patterns we observe in Fig. 6.
The usual artifacts apparent on scalp recordings, such as eye movements, body/head/tongue movements and EMG, are virtually absent with invasive recordings. The main artifacts that may be seen with intracranial EEG include pulse artifacts, if the electrode is on or near a vessel, or if the electrode wire is grossly pulled from the outside (a rare occurrence since the subdural electrodes are securely anchored). Other artifacts, such as $60 \mathrm{~Hz}$, can rarely be seen if the electrode makes poor contact with the cortical surface (e.g. from underlying fluid collection). As we stated earlier, during preprocessing ECoG recordings were visually inspected for artifact identification and rejection. Furthermore, other artifacts, such as systematic contamination from electromagnetic interference, would most probably cause smooth fields in the recordings, since the interfering source would be distant from the recordings. Therefore, such recordings would be excluded from our analysis because of our high spatial variance selection criterion.

The possibility still exists that some other reason not related to artifacts, such as fatigue, alertness, etc. might be the main cause for the difference between conditions, and not the behavioral engagement we labeled. We understand that some other internal reason might be the cause. It is indeed difficult in natural settings where isolated behaviors are studied to separate all factors. We would like, however, to point out that given the placement of the microgrid, 
Fig. 8 Permutation test statistical analysis. Top Significant voxels when comparing different pairs of conditions, corrected for multiple comparisons at a 0.05 family-wise error level. Bottom Statistical maps of original data, estimated using Eq. 5

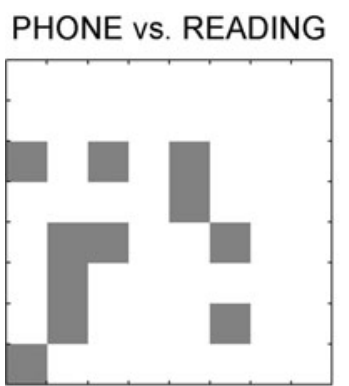

PHONE vs. FACE

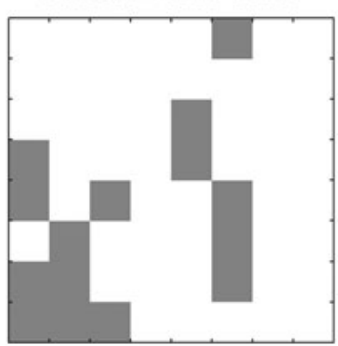

READING vs. PICTURES

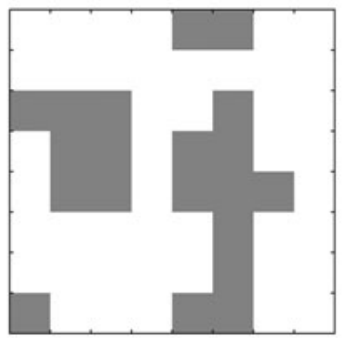

PHONE vs. PICTURES

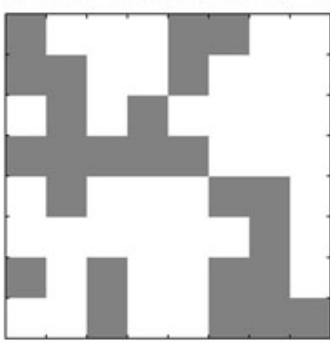

FACE vs. PICTURES
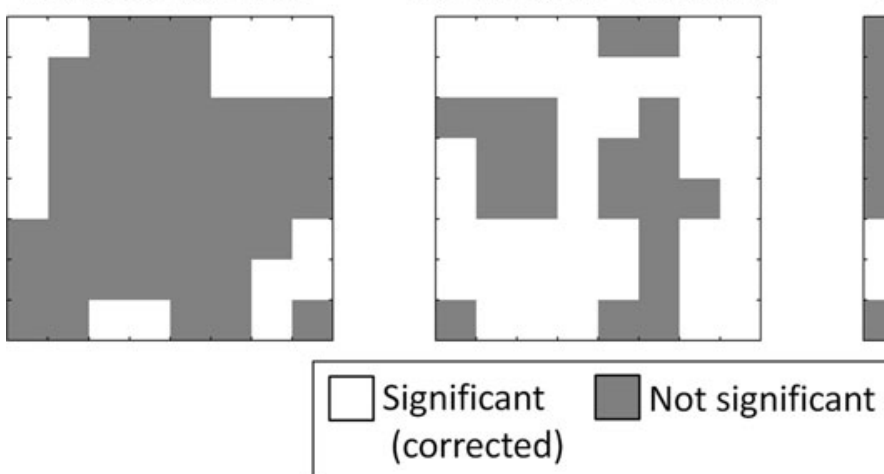

PHONE vs. READING

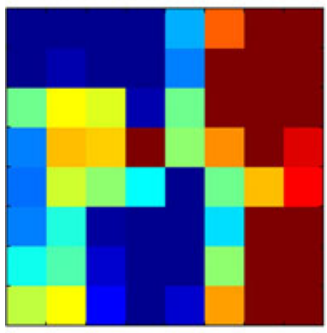

READING vs. FACE

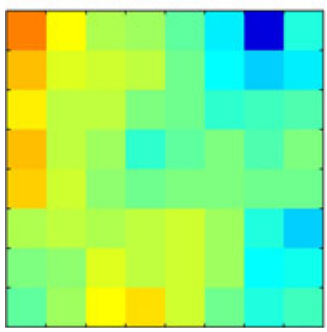

PHONE vS. PICTURES
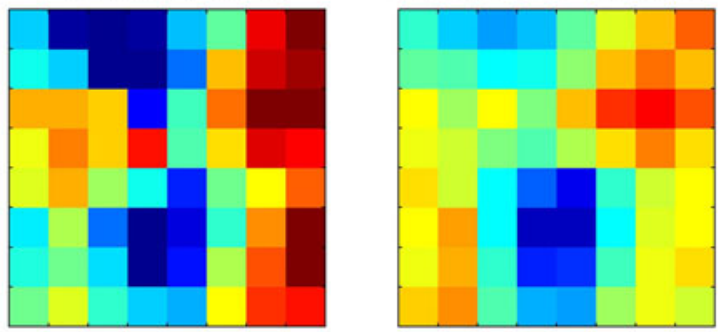

READING vs. PICTURES

FACE vs. PICTURES
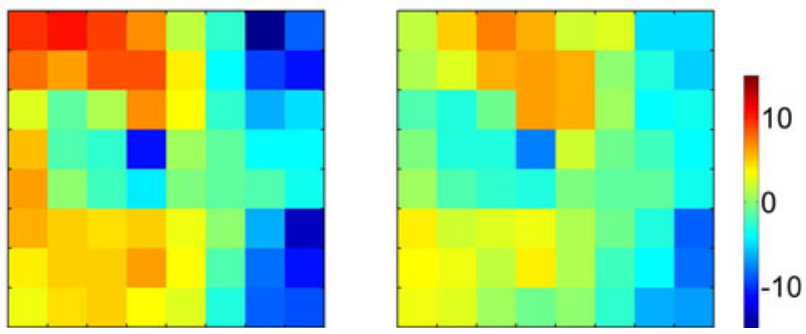

namely the right tip of the temporal lobes, such a separation by behaviors as the ones we selected would not be impossible. Furthermore, our goal is to demonstrate the existence of spatially variable signals of brain origin, even if they are not introduced by strictly defined conditions.

Our method was designed to extract high spatial frequency information from recordings of cortical activity, particularly when the microgrid was positioned on cortical areas for which behavioral correlates had already been established by conventional ECoG, MEG and fMRI methods, which by wide spacing gave low spatial frequency information. Given a limited number of electrodes and channels, there must always be a trade-off between sampling high spatial frequencies versus measuring signals from extended cortical areas. Microgrids sample high spatial frequencies but only in a small cortical area, whereas conventional ECoGs sample extended cortical areas but at the expense of limited spatial resolution and therefore aliasing.

Evidence was required that spatial textures could be detected with such close spacing, despite the smoothing of ECoG signals by volume conduction on referential recording. The evidence in animal studies came from classification of spatial patterns of amplitude modulation in the beta and gamma ranges with respect to conditioned stimuli (Freeman 
2005, 2006). This method demonstrated that, despite the shared frequencies of the 64 waveforms of the ECoG, the local differences in amplitude gave significant classificatory information. Even more significant were the spatial patterns of phase modulation, which were determined by the relative amplitudes of the Fourier components of sine and cosine. The phase gradients provided unequivocal proof of information at high spatial frequencies in the referentially recorded ECoG from animals (Freeman 2004a, b; Freeman and Barrie (2000). Very similar phase gradients from the microgrid array provided comparable proof for the preservation of amplitude information in ECoG signals in human referential recording (Freeman 2005).

The microgrid recordings utilized in this study, which provided access to "fine-grained" spatial information on human cortical EEG, resulted in findings that may also be consistent with one concept of the cortical dynamics of cognition. This theory proposes that neural activity related to perception is modulated by "cinematographic" spatiotemporal EEG patterns (Henery and Mayhew 1989). Shared cortical oscillations occurring at the same instantaneous frequency across the cortical surface may serve as a "carrier wave" for perception by means of spatial patterns (of "wave packets") of amplitude modulation (AM). The information content is broadly distributed over the cortex, not localized. Endogenous state transitions, which occur several times per second, and result in the formation a wave packet with specific AM patterns may be the essence of act of perception (Henery and Mayhew 1989; Tramo et al. 1995). We speculate that the distinct spatial patterns that we find are associated with specific behaviors in this study may in fact correspond to AM patterns of perception and intention. Future research that employs microgrid technology in other subjects will be required to better understand the dynamics of human cognition. We predict that the ECoG correlates at high spatial frequencies will be found in most if not all areas of cortex studied in subjects engaged in normal behaviors.

Acknowledgments The Authors wish to acknowledge Mr. Nickolas Noah for his assistance with editorial corrections. A preliminary report on this work has been presented (Panagiotides et al. 2008).

Open Access This article is distributed under the terms of the Creative Commons Attribution Noncommercial License which permits any noncommercial use, distribution, and reproduction in any medium, provided the original author(s) and source are credited.

\section{References}

Allison T, McCarthy G, Wood CC, Jones SJ (1991) Potentials evoked in human and monkey cerebral cortex by stimulation of the median nerve. Brain 114:2465-2503
Allison T, McCarthy G, Nobre A, Puce A, Belger A (1994) Human extrastriate visual cortex and the perception of faces, words, numbers, and colors. Cereb Cortex 4:544-554

Allison T, Puce A, Spencer DD, McCarthy G (1999) Electrophysiological studies of human face perception. I: potentials generated in occipitotemporal cortex by face and non-face stimuli. Cereb Cortex 9:415-430

Anson JA, Kuhlman DT (1993) Post-ictal Kluver-Bucy syndrome after temporal lobectomy. J Neurol Neurosurg Psychiatr 56: 311-313

Barrie JM, Freeman WJ, Lenhart M (1996) Modulation by discriminative training of spatial patterns of gamma EEG amplitude and phase in neocortex of rabbits. J Neurophysiol 76:520-539

Bucher K, Myers RE, Southwick C (1970) Anterior temporal cortex and maternal behavior in monkey. Neurology 20:415

Damasio AR, Tranel D, Damasio H (1990) Face agnosia and the neural substrates of memory. Annu Rev Neurosci 13:89-109

Duda RO, Hart PE, Stork DG (2000) Pattern classification, 2nd edn. Wiley-Interscience, New York

Freeman WJ (1975) Mass action in the nervous system. Academic Press, New York

Freeman WJ (2004a) Origin, structure, and role of background EEG activity. Part 1. Analytic amplitude. Clin Neurophysiol 115: 2077-2088. http://repositories.cdlib.org/postprints/1006

Freeman WJ (2004b) Origin, structure, and role of background EEG activity. Part 2. Analytic phase. Clin Neurophysiol 115: 2089-2107. http://repositories.cdlib.org/postprints/1486

Freeman WJ (2005) Origin, structure, and role of background EEG activity. Part 3. Neural frame classification. Clin Neurophysiol 116 (5): 1118-1129. http://authors.elsevier.com/sd/article/S1388 245705000064; http://repositories.cdlib.org/postprints/2134/

Freeman WJ (2006) Origin, structure, and role of background EEG activity. Part 4. Neural frame simulation. Clin Neurophysiol 117/ 3: 572-589. http://repositories.cdlib.org/postprints/1480/; http:// dx.doi.org/10.1016/j.clinph.2005.10.025

Freeman WJ, Barrie JM (2000) Analysis of spatial patterns of phase in neocortical gamma EEGs in rabbit. J Neurophysiol 84: $1266-1278$

Freeman WJ, Burke BC (2003) A neurobiological theory of meaning in perception. Part 4. Multicortical patterns of amplitude modulation in gamma EEG. Int J Bifurc Chaos 13: 2857-2866. http://repositories.cdlib.org/postprints/3345

Freeman WJ, Grajski KA (1987) Relation of olfactory EEG to behavior: factor analysis. Behav Neurosci 101:766-777

Freeman WJ, Rogers LJ (2002) Fine temporal resolution of analytic phase reveals episodic synchronization by state transitions in gamma EEG. J Neurophysiol 87:937-945

Freeman WJ, Viana Di Prisco G (1986) Relation of olfactory EEG to behavior: time series analysis. Behav Neurosci 100:753-763

Freeman WJ, Rogers LJ, Holmes MD, Silbergeld DL (2000) Spatial spectral analysis of human electrocorticograms including the alpha and gamma bands. J Neurosci Methods 95:111-121

Freeman WJ, Burke BC, Holmes MD, Vanhatalo S (2003a) Spatial spectra of scalp EEG and EMG from awake humans. Clin Neurophysiol 114:1053-1068

Freeman WJ, Burke BC, Holmes MD (2003b) Aperiodic phase re-setting in scalp EEG of beta-gamma oscillations by state transitions at alpha-theta rates. Hum Brain Mapp 19:248-272. http://repositories.cdlib.org/postprints/3347

Freeman WJ, Gaál G, Jornten R (2003c) A neurobiological theory of meaning in perception. Part 3. Multiple cortical areas synchronize without loss of local autonomy. Int $\mathrm{J}$ Bifurc Chaos 13: 2845-2856. http://repositories.cdlib.org/postprints/3344

Freeman WJ, Holmes MD, West GA, Vanhatalo S (2006a) Fine spatiotemporal structure of phase in human intracranial EEG. Clin Neurophysiol 117:1228-1243 
Freeman WJ, Holmes MD, West GA, Vanhatalo S (2006b) Dynamics of human neocortex that optimizes its stability and flexibility. Intern J Intell Syst 21: 1-21. http://repositories.cdlib.org/ postprints $/ 2385$

Ghovanloo MW, Wise KD, Najafi K (2003) Towards a button-sized 1024-site wireless cortical microstimulating array. Neural engineering conference proceedings, first international IEEE EMBS conference on neural engineering, pp 138-141

Glosser G, Zwil AS, Glosser DS, O'Connor MJ, Sperling MR (2000) Psychiatric aspects of temporal lobe epilepsy before and after anterior temporal lobectomy. J Neurol Neurosurg Psychiatr 68:53-58

Grill-Spector K, Knouf N, Kanwisher N (2004) The fusiform face area subserves face perception, not generic within-category identification. Nat Neurosci 7:555-562

Gross CG (1992) Representation of visual stimuli in inferior temporal cortex. Philos Trans R Soc Lond B 335:3-10

Henery CC, Mayhew TM (1989) The cerebrum and cerebellum of the fixed human brain: efficient and unbiased estimates of volumes and cortical surface areas. J Anat 167:167-180

Kanwisher N, Yovel G (2006) The fusiform face area: a cortical region specialized for the perception of faces. Philos Trans R Soc Lond B Biol Sci 361:2109-2128

Kanwisher N, McDermott J, Chun MM (1997) The fusiform face area: a module in human extrastriate cortex specialized for face perception. J Neurosci 17:4302-4311

Kanwisher N, Stanley D, Harris A (1999) The fusiform face area is selective for faces not animals. Neuroreport 10:183-187

Kling A, Steklis HD (1976) A neural substrate for affiliative behavior in nonhuman primates. Brain Behav Evol 13:216-238

Kling AS, Tachiki K, Lloyd R (1993) Neurochemical correlates of the Kluver-Bucy syndrome by in vivo microdialysis in monkey. Behav Brain Res 56:161-170

Lilly R, Cummings JL, Benson F, Frankel M (1983) The human Kluver-Bucy syndrome. Neurology 33:1141-1145

McCarthy G, Puce A, Belger A, Allison T (1999) Electrophysiological studies of human face perception. II: response properties of face-specific potentials generated in occipitotemporal cortex. Cereb Cortex 9:431-444

Mummery CJ, Patterson K, Prices CJ, Ashburner J, Frackowiak RSJ, Hodges JR (2000) A voxel-based morphometry study of semantic dementia: relationship between temporal lobe atrophy and semantic memory. Ann Neurol 47:36-45

Murai T, Fujimoto S (2003) Rapid cycling bipolar disorder after left temporal polar damage. Brain Inj 17:355-358

Nieuwenhuys R, Ten Donkelaar HJ, Nicholson C (1998) The central nervous system of vertebrates, vol. 3. Springer, Berlin

Ohl FW, Scheich H, Freeman WJ (2001) Change in pattern of ongoing cortical activity with auditory category learning. Nature 412:733-736
Olson IR, Plotzker A, Ezzyat Y (2007) The Enigmatic temporal pole: a review of findings on social and emotional processing. Brain 130:8-31

Panagiotides H, Freeman WJ, Holmes MD, Pantazis D (2008) Behavioral states exhibit distinct spatial EEG patterns. Abstr \#1.051, 62nd Ann Mtg Amer Epilepsy Soc, Seattle WA

Pang CC et al (2005) A new multi-site probe array with monolithically integrated parylene flexible cable for neural prostheses. The 27th annual international conference of the IEEE engineering in medicine and biology society (EMBS), Shanghai, China

Pantazis D, Nichols TE, Baillet S, Leahy RM (2005) A comparison of random field theory and permutation methods for the statistical analysis of MEG data. Neuroimage 25:383-394

Perrett DI, Smith PA, Potter DD, Mistlin AJ, Head AS, Milner AD et al (1985) Visual cells in the temporal cortex sensitive to face view and gaze direction. Proc R Soc B Biol Sci 223:293-317

Pockett S, Bold GEJ, Freeman WJ (2009) EEG synchrony during a perceptual-cognitive task: widespread phase synchrony at all frequencies. Clin Neurophysiol 120:695-708. doi:10.1016/ j.clinph.2008.12.044

Puce A, Allison T, McCarthy G (1999) Electrophysiological studies of human face perception. III: effects of top-down processing on face-specific potentials. Cereb Cortex 9:445-458

Reilly EL (1999) EEG recording and operation of the apparatus. In: Niedermeyer E, Lopes da Silva F (eds) Electroencephalography: basic principles, clinical applications, and related fields. Williams \& Wilkins, Baltimore, pp 122-142

Ruiz YG, Li G, Freeman WJ, Moreira EG (2007) A new approach to detect stable phase structure in high density EEG signals. 1st international conference on Cognitive Neurodynamics 2007. Shanghai, P.R. China: Springer, Chap. 128, pp 741-745

Ruiz Y, Li G, Freeman WF, Gonzalez E (2009) Detecting stable phase structures on EEG signals to classify brain activity amplitude patterns. J Zhejiang Univ 10:1483-1491

Spencer S, Sperling M, Shewmon A, Kahane P (2008) Intracranial electrodes. In: Engel J Jr, Pedley T (eds) Epilepsy: a comprehensive textbook, vol. 171. Wolters Kluwer/Lippincott, Philadelphia, pp 1791-1815

Thompson SA, Patterson K, Hodges JR (2003) Left/right asymmetry of atrophy in semantic dementia: behavioral-cognitive implications. Neurology 61:1196-1203

Tramo MJ, Loftus WC, Thomas CE, Green RL, Mott LA, Gazzaniga MS (1995) Surface area of human cerebral cortex and its gross morphological subdivisions: in vivo measurements in monozygotic twins suggest differential hemisphere effects of genetic factors. J Cogn Neurosci 7:292-302 